\title{
Quantum holonomies with Josephson-junction devices
}

\author{
Mateusz Cholascinski \\ Institut für Theoretische Festkörperphysik, Universität Karlsruhe, D-76128 Karlsruhe, Germany and \\ Nonlinear Optics Division, Adam Mickiewicz University, 61614 Poznan, Poland
}

\begin{abstract}
We examined properties of a Josephson-junction system composed of two coupled Cooper-pair boxes (charge qubits) as a candidate for observation of quantum holonomies. We construct a universal set of transformations in a twofold degenerate ground state, and discuss the effects of noise in the system.

PACS numbers: 03.67.-a, 03.65.Vf
\end{abstract}

\section{INTRODUCTION}

Appearance of the geometric phases in physical systems has been, since their first systematic treatment by Berry ${ }^{1}$, among the most fascinating physical phenomena. Generalized to systems with degenerate spectrum, ${ }^{2}$ they give the possibility to devise systems in which the dynamical contribution is only the overall phase factor, and the actual transformations are of purely geometric origin.

Quantum geometric transformations (below referred to as holonomies) have attracted even more attention after Pachos et al. ${ }^{3}$ proved their potential use in quantum computing. As compared to the ordinary, dynamical computation, quantum gates are here realized by cyclic evolution of parameters, and the result depends only on the geometry of the traversed path.

Construction of holonomies has been discussed for various physical realizations, among them are also superconducting nanocircuits. ${ }^{4,5}$ In these realizations the necessary number of independent tunable parameters results in high complexity of the considered systems. Here, motivated also by the recent experiment with Josephsonjunction system composed of two coupled charge qubits, ${ }^{6}$ we consider a similar design as a potential candidate for observation of quantum holonomies. As compared to the proposals in Refs. ${ }^{4,5}$, where the simplest two-dimensional holonomies are constructed using four coupled charge qubits, we achieve substantial simplification using only two qubits. Also, we realize the transformations within a twofold degenerate ground state, rather than excited state (as in ${ }^{4,5}$ ). In this way we avoid the problem of depopulation of the subspace in which the holonomies are realized.

We begin with describing the system and defining the operational subspace. In the four-dimensional space of two charge qubits we find a configuration of parameters for which the ground state is degenerate. We perform the holonomies by selecting two of the parameters and varying them adiabatically along a certain loop. During the transformation the degeneracy of the ground state is maintained by adjusting the remaining parameters as a function of the others.

The scheme presented here may be realized in a system without strong constraints on its parameters provided that the noise level is low. However, since the system of charge qubits is usually affected by the charge fluctuations, in the following we optimize the design in order to suppress the noise (for certain models of errors we find a decoherence-free subspace $\left.{ }^{7,8,9}\right)$. Finally we discuss possible extensions of the scheme. The specific system considered here is described by the Hamiltonian of two coupled qubits. In the discussion we also comment on the possible application of our scheme to an arbitrary system with this model Hamiltonian.

\section{THE SYSTEM}

The system we consider consists of two "charge qubits". ${ }^{10}$ They are coupled to each other via tunable Josephson junction - a symmetric dc-SQUID (superconducting quantum interference device) (see Fig. 1). As discussed later, we need to have the possibility to switch off all the Josephson energies completely (and we use dc-SQUIDS instead of simple junctions). On the other hand, to construct nontrivial transformations, the amplitude and phase (or equivalently the real and imaginary parts) of the couplings $J_{1}$ and $J_{2}$ should be controllable during the operations. To achieve this we replace one of the junctions in the left and right SQUIDs with a further dc-SQUIDs (the same technique has been used in Ref. ${ }^{4}$ ).

If the dimensionless gate charges $n_{g 1(2)}=$ $C_{g 1(2)} V_{g 1(2)} / 2 e$ are close to $1 / 2$, the only relevant charge states for each island are $|0\rangle$ and $|1\rangle$ (zero or one extra Cooper pair on the island). The Hamiltonian may be then reduced to the four-dimensional space and in the charge basis $\left|n_{1}, n_{2}\right\rangle$ has the form

$$
H=\left(\begin{array}{cccc}
E_{00} & -J_{2} / 2 & -J_{1} / 2 & 0 \\
-J_{2}^{*} / 2 & E_{01} & -J_{m} / 2 & -J_{1} / 2 \\
-J_{1}^{*} / 2 & -J_{m} / 2 & E_{10} & -J_{2} / 2 \\
0 & -J_{1}^{*} / 2 & -J_{2}^{*} / 2 & E_{11}
\end{array}\right) .
$$

Here the diagonal elements are the electrostatic terms $E_{n 1, n 2}=E_{c 1}\left(n_{g 1}-n_{1}\right)^{2}+E_{c 2}\left(n_{g 2}-n_{2}\right)^{2}+$ $E_{m}\left(n_{g 1}-n_{1}\right)\left(n_{g 2}-n_{2}\right) \quad\left[n_{1}\left(n_{2}\right)\right.$ is the number of excess Cooper pairs on the first (second) island] with $E_{c 1}=4 e^{2} C_{\Sigma 2} / 2\left(C_{\Sigma 1} C_{\Sigma 2}-C_{m}^{2}\right)$, and similarly for $E_{c 2}$. Here $C_{\Sigma 1(2)}$ is the sum of all capacitances connected to the first (second) island. The electrostatic coupling 


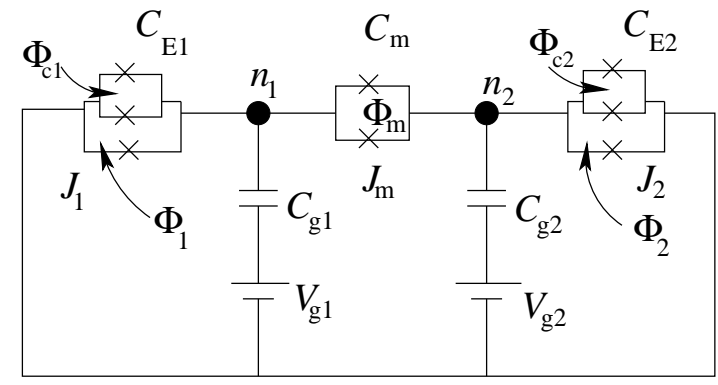

FIG. 1: The system of two coupled charge qubits used to construct the simplest non-Abelian holonomies. The superconducting islands ("Cooper-pair boxes") are denoted by black nodes. $n_{1}$ and $n_{2}$ are the numbers of excess Cooper pairs on the islands. The Josephson coupling between the two islands may be turned off completely. The couplings $J_{1}$ and $J_{2}$ can be on one hand turned off completely, on the other hand we may obtain complex couplings by changing the fluxes $\Phi_{c 1(2)}$.

term due to finite capacitance of the middle dc-SQUID equals $E_{m}=4 e^{2} C_{m} /\left(C_{\Sigma 1} C_{\Sigma 2}-C_{m}^{2}\right.$ ) (we do not assume weak electrostatic coupling and the capacitance $C_{m}$ does not need to be small). The Josephson coupling $J^{(1)}=$ $\sqrt{\left(J_{j l}^{(1)}-J_{j r}^{(1)}\right)^{2}+4 J_{j l}^{(1)} J_{j r}^{(1)} \cos ^{2}\left(\pi \Phi_{1}\right)} \exp \left[-i \psi\left(\Phi_{1}\right)\right]$ (and similarly for $\left.J^{(2)}\right)$, where $\tan \psi\left(\Phi_{1}\right)=$ $\left(J_{j l}^{(1)}-J_{j r}^{(1)}\right) /\left(J_{j l}^{(1)}+J_{j r}^{(1)}\right) \tan \pi \Phi_{1} \cdot{ }^{11}$ Here $J_{j l}$ is the Josephson energy of the junctions in the dc-SQUIDs. The term $J_{j r}=J_{j r(0)} \cos \pi \Phi_{c}$ is the tunable Josephson coupling of the small SQUID. Finally $J_{m}=J_{m}^{(0)} \cos \pi \Phi_{m}$ is the Josephson energy of the middle SQUID. Here the fluxes are in units of $\Phi_{0}=h c / 2 e$, the superconducting flux quantum. We assume that the system is operated in the charge regime $\left(E_{c 1(2)}, E_{m} \gg J_{j l(r)}^{(1)}, J_{j l(r)}^{(2)}, J_{m}^{(0)}\right)$.

A similar system has been used already in the context of geometric phases. Falci et al. ${ }^{12}$ used two coupled charge qubits to devise an experiment in which the Berry phase could be detected. Also, as already mentioned, the experiment by Pashkin et al. ${ }^{6}$ shows that a system of two coupled charge qubits can be well controlled and manipulated with available experimental techniques. The difference between our system and those used in Refs. ${ }^{6,12}$ is that we couple the islands using dc-SQUID instead of a capacitor, and we require controllable Josephson couplings $J_{1(2)}$.

The set of controllable parameters defines the control manifold. The Hamiltonian is characterized by the tunable terms: $E_{c 1}, E_{c 2}, J_{1}, J_{2}, J_{m}$. We will use in the discussion rather the parameters that can be controlled directly, i.e., $\left(\Phi_{1}, \Phi_{2}, \Phi_{m}, n_{g 1}, n_{g 2}\right)$. The fluxes $\Phi_{c 1(2)}$ are used only to control the symmetry of the SQUIDs, and we will not consider them to be used as the actual tunable parameters in the following discussion (between the operations they are kept at the values for which the couplings $J_{1(2)}$ are real, and may be tuned to zero. Just before the operations we can switch them to different values for which the dc-SQUIDs are asymmetric. During the operations they are kept constant).

The operational subspace for the simplest non-Abelian holonomies is spanned by two lowest-energy eigenstates. To make the transformations of purely geometric nature we need to make them degenerate. This property in our system does not follow from the symmetry arguments and we need to control it by making the parameters not fully independent. In the following we will compare the energies of the lowest states, and from this constraint calculate one of the parameters as the function of the remaining ones. In this way we define the degeneracy domain in the control manifold.

For the following configuration of the parameters

$$
J_{m}=J_{1}=J_{2}=0, \quad n_{g 1}=n_{g 2}=1 / 2,
$$

the Hamiltonian Eq.(1) is diagonal in the charge basis, and the ground state is twofold degenerate and spanned by the states

$$
|\overline{0}\rangle \equiv|01\rangle, \quad|\overline{1}\rangle \equiv|10\rangle .
$$

We will refer to this particular point in the parameter space as the starting point, or the "no-op" regime (as this configuration will be kept between the sequences of transformations), and to the states $|\overline{0}\rangle$ and $|\overline{1}\rangle$ as the logical basis.

Before we perform any transformations the system is initialized to the state $|\overline{0}\rangle$ by turning off all the Josephson couplings, and tuning the electrostatic energy $E_{01}$ to be the lowest one. After long enough time the system will relax, and we switch to the starting point. We perform a holonomic transformation by varying the parameters adiabatically along a suitable loop. During the operations the other charge states may be involved as well. The system, however, at any instant of time remains in the twofold degenerate ground state, and the resulting unitary transformation (holonomy) is limited to the logical basis only. The correspondence between the loop, and the performed unitary transformation is given by

$$
U_{\Gamma}=\mathcal{P} \exp \left(-\oint_{\Gamma} \sum_{i} \mathcal{A}_{i} d X_{i}\right),
$$

where $\mathcal{P}$ is the path-ordering operator, $\Gamma$ the path, $R=$ $\left\{X_{i}\right\}$ is the set of parameters (control manifold), and the $2 \times 2$ matrix $\mathcal{A}_{i}$ is the Wilczek-Zee connection, ${ }^{2}$

$$
\mathcal{A}_{i}^{\alpha \beta}=\left\langle\alpha(R)\left|\frac{\partial}{\partial X_{i}}\right| \beta(R)\right\rangle .
$$

$|\alpha\rangle$ and $|\beta\rangle$ are parameter-dependent states spanning the operational subspace, and for the 'no-op' regime they can be either $|\overline{0}\rangle$ or $|\overline{1}\rangle$.

To construct the holonomies we need to find a loop corresponding to the desired transformation. Any unitary transformation can be factorized as a product of two noncommuting rotations in the qubit space. Here we find two such holonomies, namely, $\exp \left(i \phi_{1} \sigma_{z}\right)$ and 
$\exp \left(i \phi_{2} \sigma_{x}\right)$. For our purposes it is convenient to interpret these rotations as phase shifts between $|\overline{0}\rangle$ and $|\overline{1}\rangle$, and between $|+\rangle=(|\overline{0}\rangle+|\overline{1}\rangle) / \sqrt{2}$ and $|-\rangle=(|\overline{0}\rangle-|\overline{1}\rangle) / \sqrt{2}$, respectively. For given rotation, at each point of the constructed paths the Wilczek-Zee connection $\mathcal{A}$ is then diagonal [in the basis $|\overline{0}(\overline{1})\rangle$ and $|+(-)\rangle$ respectively], and we omit the path-ordering operation.

\section{CONSTRUCTING THE HOLONOMIES}

The holonomies we find are in our case the aforementioned rotations, $e^{i \phi_{1} \sigma_{z}}$ and $e^{i \phi_{2} \sigma_{x}}$. The first transformation we consider is the phase shift $e^{i \phi_{1} \sigma_{z}}$. If we keep $J_{1}=J_{m} \equiv 0$ (and change the flux $\Phi_{c 2}$ to make the coupling $J_{2}$ complex), the Hamiltonian simplifies to

$$
H_{1}=\left(\begin{array}{cccc}
E_{00} & -J_{2} / 2 & 0 & 0 \\
-J_{2}^{*} / 2 & E_{01} & 0 & 0 \\
0 & 0 & E_{10} & -J_{2} / 2 \\
0 & 0 & -J_{2}^{*} / 2 & E_{11}
\end{array}\right) .
$$

The obtained block form may be solved for each part independently. We calculate the desired phase shift as the difference between the Berry phases for each block. At the same time, to make the phase shift of purely geometric origin, we compare the energies of the lower states within each block to find the degeneracy subspace in the control manifold. Since the energies of the lowest states are functions of $n_{g 1}, n_{g 2}$, and $\Phi_{2}$, from the condition $E_{\alpha}\left(n_{g 1}, n_{g 2}, \Phi_{2}\right)-E_{\beta}\left(n_{g 1}, n_{g 2}, \Phi_{2}\right)=0$ we find the degeneracy condition in the functional form $n_{g 1}\left(n_{g 2}, \Phi_{2}\right)$. This dependence is shown in the upper part of Fig. 2

The parameter-dependent spectrum (for the degeneracy domain in the control manifold) is shown in the middle plot of Fig. 2. The effectively four-level system has only three distinct energy values due to the degeneracy of the ground state.

Finally the phase shift may be presented conveniently as the integral,

$$
\phi_{1}=-\frac{1}{2} \int_{S(\Gamma)} d n_{g 2} d \Phi_{2}\left[V_{\alpha}\left(n_{g 2}, \Phi_{2}\right)-V_{\beta}\left(n_{g 2}, \Phi_{2}\right)\right],
$$

where $S(\Gamma)$ is the domain in the $n_{g 2}-\Phi_{2}$ plane limited by the path $\Gamma$, and the "Berry field" is given by

$$
V_{\alpha(\beta)}=-i \frac{\left\langle\alpha(\beta)\left|\nabla_{R} H_{1}\right| e_{a}\left(e_{b}\right)\right\rangle \times\left\langle e_{a}\left(e_{b}\right)\left|\nabla_{R} H_{1}\right| \alpha(\beta)\right\rangle}{\left(E_{\alpha(\beta)}-E_{e a(e b)}\right)^{2}} .
$$

Here $\left|e_{a}\right\rangle$ and $\left|e_{b}\right\rangle$ are the excited states from the upperleft and the lower-right block of $H_{1}$ respectively, and $E_{e a(e b)}$ their energies. The field difference $V=V_{\alpha}-V_{\beta}$ is shown in the lower plot of Fig. 2.

Similarly, we can find the second generic holonomy, namely, $e^{i \phi_{2} \sigma_{x}}$. For $J_{1}=J_{2} \equiv J$ (the coupling should be complex during the operations, so the fluxes $\Phi_{c 1(2)}$ should have different values than at the starting point, but chosen in this way that the Josephson energies may
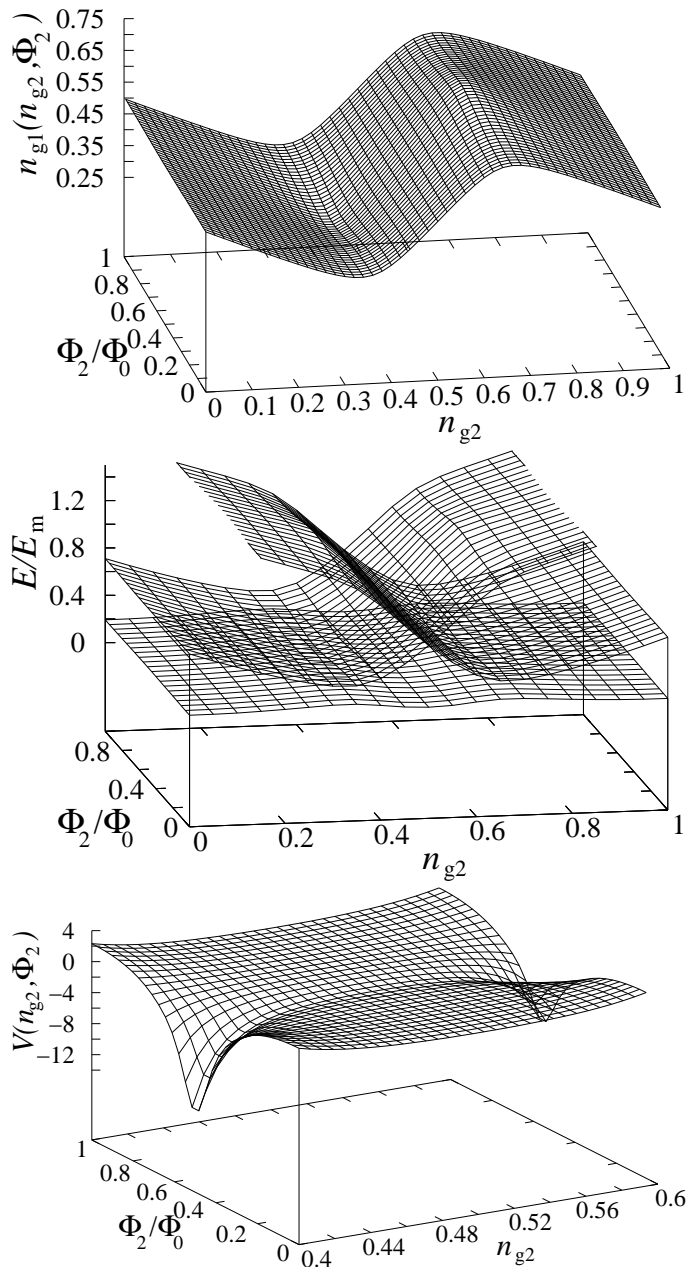

FIG. 2: Construction of the $\exp \left(i \phi_{1} \sigma_{z}\right)$ rotation. Upper plot - the degeneracy subspace calculated from the comparison of the energies of the lowest-energy levels; middle plot - the parameter-dependent spectrum of the system (the ground state is twofold degenerate); lower plot - the "Berry field". All plots taken for the following values of system parameters: $E_{c 1} / E_{m}=0.8, E_{c 2} / E_{m}=1, J_{r}^{2} / E_{m}=0.1$, and $J_{l}^{2} / E_{m}=0.05$.

be equal), $E_{01}=E_{10} \equiv E$, the Hamiltonian may be again presented in the block form, this time in the basis $\{|00\rangle,|11\rangle,| \pm\rangle=(|01\rangle \pm|10\rangle) / \sqrt{2}\}$ :

$$
H_{2}=\left(\begin{array}{cccc}
E_{00} & 0 & -\frac{J}{\sqrt{2}} & 0 \\
0 & E_{11} & -\frac{J}{\sqrt{2}} & 0 \\
-\frac{J^{*}}{\sqrt{2}} & -\frac{J^{*}}{\sqrt{2}} & E-J_{m} / 2 & 0 \\
0 & 0 & 0 & E+J_{m} / 2
\end{array}\right) .
$$

Comparison of the lowest energy of the upper-left block with $E+J_{m} / 2$ gives the degeneracy subspace, as shown in the upper plot of Fig. 3. The middle plot shows the parameter-dependent spectrum, again the ground state being twofold degenerate. Since the state $|-\rangle$ does not vary with the parameters, the desired phase is simply the Berry phase acquired by the ground state in the upper- 
left block. The phase here equals

$$
\phi_{2}=\frac{1}{2} \int_{S(\Gamma)} d n_{g 2} d \Phi V^{\prime}\left(n_{g 2}, \Phi\right) .
$$

The field $V^{\prime}$ is shown in the lower plot of Fig. 3 .
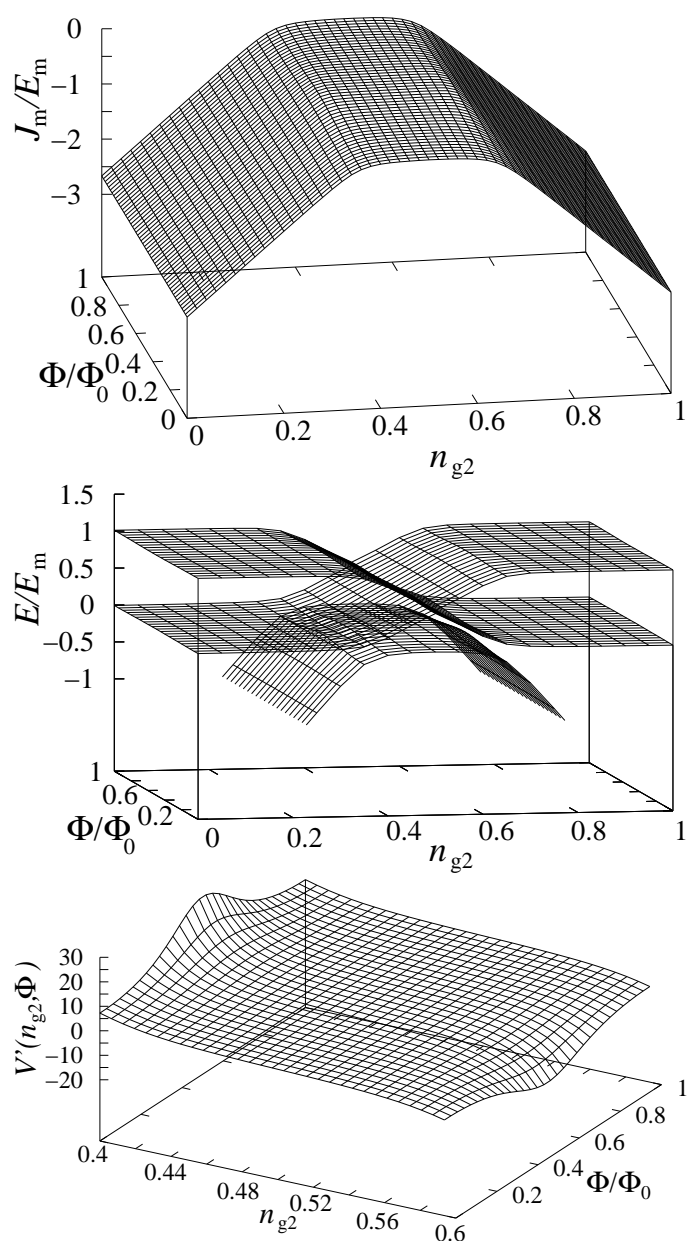

FIG. 3: Construction of the $\exp \left(i \phi_{2} \sigma_{x}\right)$ rotation. Upper plot - the degeneracy subspace calculated from the comparison of the energies of the lowest-energy levels; middle plot - the parameter-dependent spectrum of the system (the ground state is twofold degenerate); lower plot - the "Berry field". All plots taken for the following values of system parameters: $E_{c 1} / E_{m}=0.8, E_{c 2} / E_{m}=1, J_{r}^{1(2)} / E_{m}=0.1$, and $J_{l}^{1(2)} / E_{m}=0.05$. Here we assumed for simplicity that the dcSQUIDs are identical so that $J_{1}=J_{2}$ implies $\Phi_{1}=\Phi_{2}=\Phi$. In practice only the first condition needs to be satisfied

\section{DISSIPATIVE EFFECTS}

The scheme described above is valid for a system without any strong constraints on its parameters - the only idealization so far is the assumption of perfect symmetry of the small dc-SQUIDs. As already noted, the operations are performed within a ground state, and the relaxation does not depopulate the operational subspace.
However, as the degeneracy is maintained by external parameters, we should expect that the system will suffer from their fluctuations. Now we will give qualitative analysis of the noise in the considered system, and describe how the design can be optimized in order to suppress the dissipation. In Josephson charge qubits the Ohmic noise and the low-frequency charge fluctuations coming from the substrate are usually relatively strong. Since the system is operated in the charge regime, and both sources couple to the charge on the islands we apply here the spin-boson model with the system Hamiltonian approximated by its electrostatic part only

$$
\begin{aligned}
H= & -\frac{\omega_{1}}{2} \sigma_{z}^{1}-\frac{\omega_{1}}{2} \sigma_{z}^{2}+J \sigma_{z}^{1} \sigma_{z}^{2}+\sum_{k} \omega_{k} a_{k}^{\dagger} a_{k} \\
& +\left(\sigma_{z}^{1}+\sigma_{z}^{2}\right) X+\sigma_{z}^{1} Y_{1}+\sigma_{z}^{2} Y_{2} .
\end{aligned}
$$

Here $X, Y_{i}$ are the bath operators. We divided the interaction term into the part describing the common environment for the two islands, and part with independent environments. If now the two islands have equal energy splitting $\left(\omega_{1}=\omega_{2} \equiv \omega_{0}\right)$, and the dominant interaction with environment can be modeled by common environment, the subspace $\{|01\rangle,|10\rangle\}$ is well protected (in the limiting case of completely correlated noise $Y_{i} \equiv 0$ the states span the decoherence-free subspace). Our goal is then to minimize the uncorrelated noise in the islands as much as possible. To achieve this for the noise coming from the substrate we can, as discussed in Ref. ${ }^{13}$ place the islands close to each other. To suppress the Ohmic noise we could use a common voltage source controlling the bias of the islands (as in the experiment by Pashkin et al. ${ }^{6}$ ). As we present here, this may work well also for our purposes, provided that the islands are nearly identical. We use again two voltage sources, but now their roles are not symmetric. Instead, the main, common source (the fluctuations of which give correlated noise) will be used to tune the system, while the second, auxiliary, is connected to one island via very small capacitance (compared to $C_{g 1(2)}$ ) as it is used to tune a small (as explained below) difference between the gate charges $n_{g 1}$ and $n_{g 2}$. The resulting noncorrelated noise will be then much lower than the correlated noise of the main source.

To be more specific, the fluctuations in $n_{g}$ should be correlated (rather than in $V_{g}$ ) and we require that the electrostatic properties of both islands are nearly identical $\left(\left|E_{c 1}-E_{c 2}\right|=\delta E_{c} \ll E_{c 1(2)}\right.$, and $C_{g 1}-C_{g 2}=\delta C_{g} \ll$ $\left.C_{g 1(2)}\right)$. Then the common voltage source $V_{g 1}=V_{g 2}$ gives first of all nearly equal energy splitting of the islands [ $\omega_{1} \approx \omega_{2}$ in Eq.(11)], and also relates the parameters $n_{g 1} \approx n_{g 2}$. We note that for the starting point as well as during the operation $e^{i \phi_{2} \sigma_{x}}$ we have $E_{01}=E_{10}$. This for identical islands gives $n_{g 1}=n_{g 2}$. Also during the operation $e^{i \phi_{1} \sigma_{z}}$ there is a region in the parameter space in which the relation $n_{g 1}=n_{g 1}\left(n_{g 2}, \Phi_{2}\right)$ nearly coincides with $n_{g 1}=n_{g 2}$ [this region is determined by $E_{01}=E_{10}\left\langle E_{00}, E_{11}\right.$, where the states $\left.\mid \overline{0}\right\rangle$ and $|\overline{1}\rangle$ have equal and lowest electrostatic energies. The small difference between $n_{g 2}$ and $n_{g 1}\left(n_{g 2}, \Phi_{2}\right)$ is in this region 
a result of small, but nonzero Josephson energies]. We then can use an additional voltage source that is coupled only to the first island. This voltage will give an additional term $\delta n_{g 1}$ which compensates the effect of small differences in the properties of the islands, and during the $e^{i \phi_{1} \sigma_{z}}$ operation the small difference $n_{g 2}-n_{g 1}\left(n_{g 2}, \Phi_{2}\right)$.

Since each gate can be performed using various paths, we may even further improve the fidelity of the operations by examining the sensitivity of the degeneracy splitting to the fluctuations of each parameter, and choose paths from the region least affected by the fluctuations. For instance, close to the starting point [defined in Eq.(2)] the main voltage $V_{g}$ fluctuations to the first order do not shift the system out of the degeneracy subspace. The same applies to $\Phi_{2}$ for the $e^{i \phi_{1} \sigma_{z}}$ operation and to $\Phi$ for $e^{i \phi_{2} \sigma_{x}}$. This can be easily seen in the upper plots of Figs. 2 and 3. Those fluctuations lie within a plane tangential to the degeneracy subspace. To protect the system also during the operation we should construct the loops relatively close to the starting point.

\section{DISCUSSION}

To summarize, we have found that the system properties give us the opportunity to realize any unitary transformation within a qubit space using gates of purely geometric origin. Assuming the perfect performance not affected by the noise we may rely on the simplest design without strong constraints on the system parameters. However, such a system composed of two solidstate qubits is usually strongly probed by the environment which results in fast dephasing. Our qualitative analysis made for this particular system specifies the conditions under which the system should be well protected even in the presence of noise.

We have not discussed here the coupling between such elementary blocks, as the question of applicability of holonomies in quantum computing remains open and requires detailed analysis of resulting fault tolerance for individual proposals. However, as the logical basis here is defined by states differing by the charge configuration of the islands, such extension based on the method described in Ref. ${ }^{4}$ should be adjustable to our system.

The scheme presented here may be applied to any system described by the Hamiltonian of two interacting qubits of the form

$$
H=B_{1} \cdot \sigma^{1}+B_{2} \cdot \sigma^{2}+J_{z} \sigma_{z}^{1} \sigma_{z}^{2}+J_{\perp}\left(\sigma_{+}^{1} \sigma_{-}^{2}+\text { h.c. }\right),
$$

provided the parameters are tunable. Since there are until now many well-developed schemes concerning qubit control as well as the controlled qubit-qubit coupling (usually for the sake of quantum computation), realization of quantum holonomies may appear very natural and straightforward in such systems. For the same reason the model of a four-dimensional quantum system as a candidate to construct and observe holonomies may turn out to be more applicable than the simplest, and widely used in implementation-independent discussions three-level model (see for example ${ }^{3}$ ).

As far as the Josephson-junction systems are considered, we may also use the device to study the process of the adiabatic charge transport: ${ }^{14}$ the logical basis is spanned by the states with one excess Cooper-pair on one island and the second with zero. Quite naturally the second discussed operation, generated by $\sigma_{x}$, corresponds for $\phi_{2}=\pi / 2$ to the charge pumping cycle. ${ }^{4}$

\section{Acknowledgments}

The author thanks $\mathrm{Yu}$. Makhlin for inspiring discussions and numerous comments on the manuscript, R. Fazio, J. Siewert, and G. Falci for discussions and useful hints. This work was supported by the DFG-Schwerpunktprogramm "QuantenInformationsverarbeitung", EU IST Project SQUBIT, and EC Research Training Network.
1 M. V. Berry, Proc. R. Soc. London, Ser. A 392, 45 (1984).

${ }^{2}$ F. Wilczek and A. Zee, Phys. Rev. Lett. 52, 2111 (1984).

3 J. Pachos, P. Zanardi, and M. Rasetti, Phys. Rev. A 61, 010305(R) (2000); P. Zanardi and M. Rasetti, Phys. Lett. A 264, 94 (1999).

${ }^{4}$ L. Faoro, J. Siewert, and R. Fazio, Phys. Rev. Lett. 90, 028301 (2003).

5 M. -S. Choi, J. Phys.: Condens. Matt. 15(46), 7823 (2003).

${ }^{6}$ Yu. A. Pashkin, T. Yamamoto, O. Astafiev, Y. Nakamura, D. V. Averin, and J. S. Tsai, Nature (London) 421, 823 (2003).

7 L.-M. Duan and G.-C. Guo, Phys. Rev. Lett. 79, 1953 (1997).

8 P. Zanardi and M. Rasetti, Phys. Rev. Lett. 79, 3306
(1997).

9 D. A. Lidar, I. L. Chuang, and K. B. Whaley, Phys. Rev. Lett. 81, 2594 (1998).

$10 \mathrm{Yu}$. Makhlin, G. Schön, and A. Shnirman, Nature (London) 398, 305 (1999).

11 M. Tinkham, Introduction to Superconductivity, 2nd ed. (McGraw-Hill, New York, 1996).

12 G. Falci, R. Fazio, G. M. Palma, J. Siewert, and V. Vedral, Nature (London) 407, 355 (2000).

13 A. B. Zorin, F.-J. Ahlers, J. Niemeyer, T. Weimann, and H. Wolf, Phys. Rev. B 53, 13682 (1996).

14 J. P. Pekola, J. J. Toppari, M. Aunola, M. T. Savolainen, and D. V. Averin, Phys. Rev. B 60, 9931 (1999). 